\title{
Crystal Structure and Mechanistic Molecular Modeling Studies of Mycobacterium tuberculosis Diterpene Cyclase Rv3377c
}

\section{Supplementary information}

Yue Zhang ${ }^{1}$, Lisa M. Prach*,2, Terrence E. O’Brien ${ }^{1}$, Frank Dimaio ${ }^{3}$, Daniil M. Prigozhin ${ }^{6}$, Jacob E. Corn ${ }^{5}$, Tom Alber ${ }^{6}$, Justin B. Siegel*,1,7,8, Dean J. Tantillo*,1

${ }^{1}$ Department of Chemistry, University of California-Davis, Davis, CA 95616, United States;

${ }^{2}$ Department of Molecular and Cell Biology, University of California, Berkeley, CA 94720;

${ }^{3}$ Department of Biochemistry, University of Washington, Seattle WA 98195;

${ }^{4}$ Molecular Biophysics and Integrated Bioimaging Division, Lawrence Berkeley National Laboratory, Berkeley, CA 94720, USA;

${ }^{5}$ Department of Biology, ETH Zurich, 8093 Zurich Switzerland;

${ }^{6}$ Department of Molecular \& Cell Biology and QB3 Institute, University of California, Berkeley, CA 94720;

${ }^{7}$ Department of Biochemistry and Molecular Medicine, University of California-Davis, Davis, CA 95616, United States;

${ }^{8}$ Genome Center, University of California-Davis, Davis, CA 95616, United States

*Corresponding authors: jbsiegel@ucdavis.edu; djtantillo@ucdavis.edu, lisamprach@,gmail.com 


\section{Table of Contents}

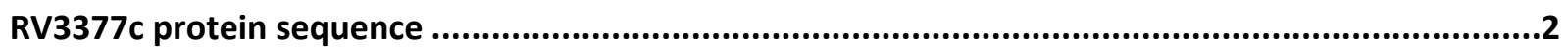

Active site volume

Complete pathway from GGPP to 1-tuberculosinyladenosine ....................................................5

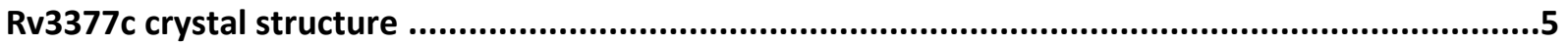

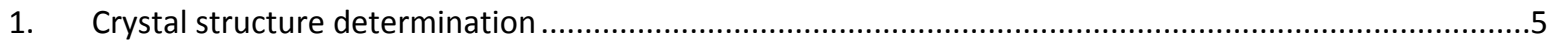

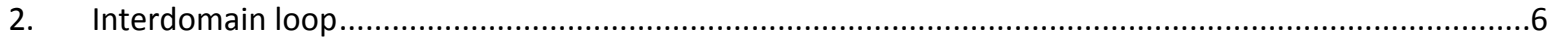

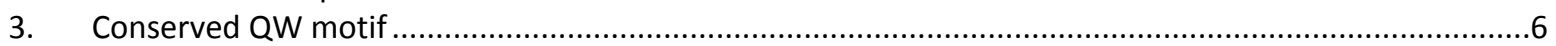

Electronic structure calculation (Density functional theory) .......................................................

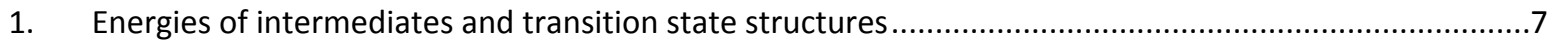

2. Intermediates and transition state structures ...............................................................................

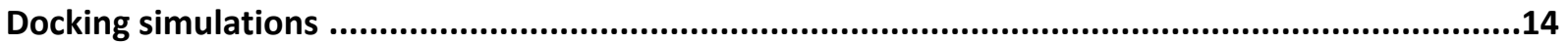

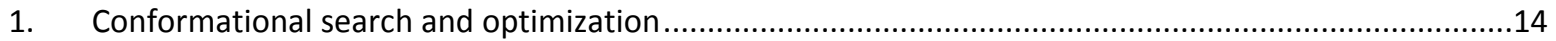

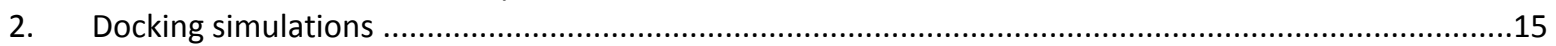

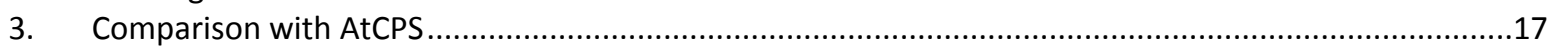

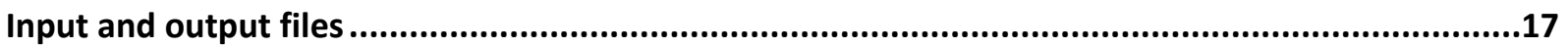

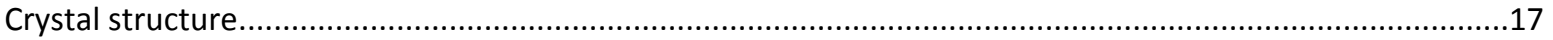

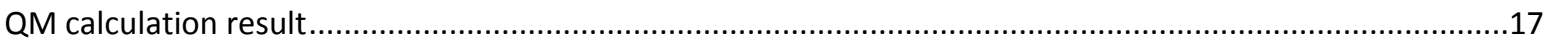

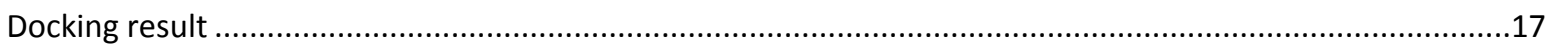

RV3377c protein sequence

$>$ Rv3377c Transcript

METFRTLLAKAALGNGISSTAYDTAWVAKLGQLDDELSDLALNWLCERQLPDGSWGA EFP

FCYEDRLLSTLAAMISLTSNKHRRRRAAQVEKGLLALKNLTSGAFEGPQLDIKDATVGF $\mathrm{E}$

LIAPTLMAEAARLGLAICHEESILGELVGVREQKLRKLGGSKINKHITAAFSVELAGQDG

VGMLDVDNLQETNGSVKYSPSASAYFALHVKPGDKRALAYISSIIQAGDGGAPAFYQAE I

FEIVWSLWNLSRTDIDLSDPEIVRTYLPYLDHVEQHWVRGRGVGWTGNSTLEDCDTTSV A

YDVLSKFGRSPDIGAVLQFEDADWFRTYFHEVGPSISTNVHVLGALKQAGYDKCHPRV RK

VLEFIRSSKEPGRFCWRDKWHRSAYYTTAHLICAASNYDDALCSDAIGWILNTQRPDGS W

GFFDGQATAEETAYCIQALAHWQRHSGTSLSAQISRAGGWLSQHCEPPYAPLWIAKTLY $\mathrm{C}$ 
SATVVKAAILSALRLVDESNQ

Highlighted residues are not in model (residues 1, 103-114 [these are residues in an interdomain loop], 139-143, and 501)

QW motifs (Q at residue 49, 190, 414, 453)

40 ALNWLCERQLPDGSW 55 (Q and W stack like reported; in a loop region)

405 AIGWILNTQRPDGSW 420 (Q and W stack like reported; in a loop region)

Bold=classic QW motif elements

Active site (D294, D295, T296)

$>$ Rv3377c nucleic acid sequence

ATGGAGACTTTCAGGACTCTGCTAGCGAAAGCGGCACTAGGCAACGGCATCTCGAG CACA

GCCTATGACACCGCGTGGGTCGCGAAGCTGGGCCAGCTCGATGATGAGCTAAGCGA CCTC

GCCCTCAACTGGCTTTGCGAGCGCCAGCTTCCTGACGGCTCATGGGGAGCCGAGTTC CCT

TTCTGCTACGAAGATCGACTGCTCTCCACTTTGGCGGCCATGATCAGCCTCACGTCG AAT

AAGCATCGTCGGAGGCGCGCAGCGCAAGTTGAAAAAGGCTTGCTAGCCCTGAAGAA CCTT

ACCTCGGGTGCTTTCGAAGGCCCTCAACTTGATATAAAAGACGCAACCGTCGGGTTT GAG

TTAATCGCACCTACGCTGATGGCTGAGGCGGCGCGACTTGGCCTTGCCATTTGCCAC GAG

GAATCCATCCTCGGCGAGCTTGTCGGAGTGCGTGAGCAGAAGTTGAGAAAACTCGG CGGA

AGCAAAATCAATAAACATATCACCGCAGCTTTCTCCGTAGAACTGGCCGGCCAAGA CGGT

GTCGGTATGTTGGATGTCGATAACTTGCAGGAGACAAATGGATCGGTCAAGTATTCG CCA

TCAGCGTCAGCCTACTTCGCGTTACATGTTAAACCAGGAGACAAGCGGGCCTTGGCG TAC

ATCTCATCGATAATTCAAGCCGGCGATGGCGGTGCGCCGGCCTTCTATCAAGCCGAG ATT

TTTGAAATTGTTTGGTCTCTATGGAATCTCTCGCGTACGGATATTGATTTGTCCGATC CT

GAGATCGTTCGTACGTACCTACCCTATCTTGACCATGTCGAACAACATTGGGTCCGT GGT

CGAGGTGTGGGGTGGACAGGAAATTCCACCTTGGAAGATTGTGACACCACAAGTGT GGCC

TACGATGTTTTGTCGAAGTTTGGACGGTCGCCGGATATCGGAGCCGTATTACAATTC GAA

GACGCCGATTGGTTCCGTACCTACTTTCACGAAGTCGGCCCCTCGATATCGACGAAC GTC

CACGTGCTCGGTGCGTTGAAGCAGGCGGGCTACGACAAATGTCATCCACGAGTACG AAAG 
GTTCTGGAATTCATCCGATCAAGCAAGGAGCCTGGACGGTTCTGCTGGCGGGATAA GTGG

CACCGATCGGCATATTATACGACTGCGCATCTGATATGTGCTGCCAGTAACTATGAT GAT

GCCCTATGCTCAGATGCGATTGGGTGGATTCTTAATACGCAGAGGCCCGATGGCTCG TGG

GGATTTTTCGACGGCCAAGCGACTGCGGAAGAGACAGCATATTGCATTCAAGCTCTG GCG

CATTGGCAGAGGCACAGCGGCACATCCTTGTCGGCGCAGATCAGTCGCGCGGGTGG GTGG

CTATCGCAACACTGCGAACCGCCATACGCGCCGTTGTGGATTGCCAAGACACTTTAC TGC

TCGGCGACGGTAGTTAAGGCTGCCATCCTAAGTGCACTGCGGCTGGTCGATGAGAGT AAC CAATGA

Active site volume

The active site volume is measured by CAVER analyst, with probe: $1.4 \AA$ radius. The volume is $738.4 \AA^{3}$. The orange sphere indicates the active site cavity.

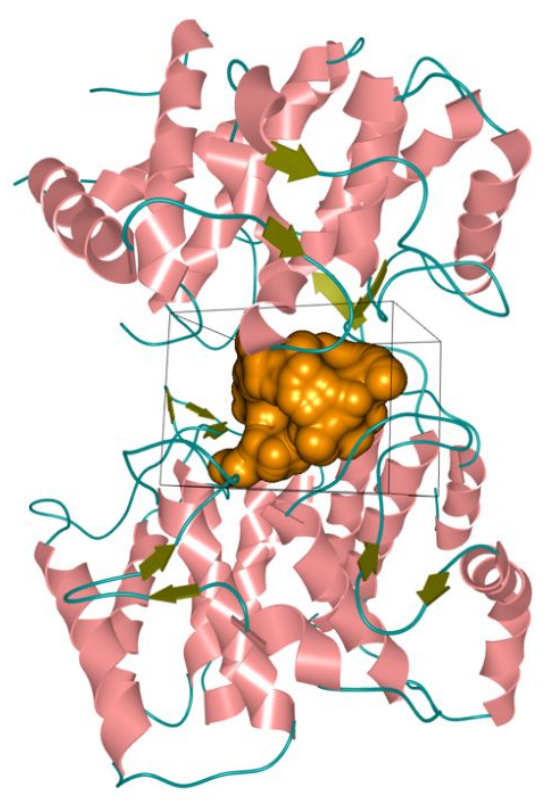

Figure s1. Active site cavity. The active site between two domains are shown in orange. Active site volume is $738.4 \AA^{3}$. Measured by CAVER analyst. 
Complete pathway from GGPP to 1-tuberculosinyladenosine
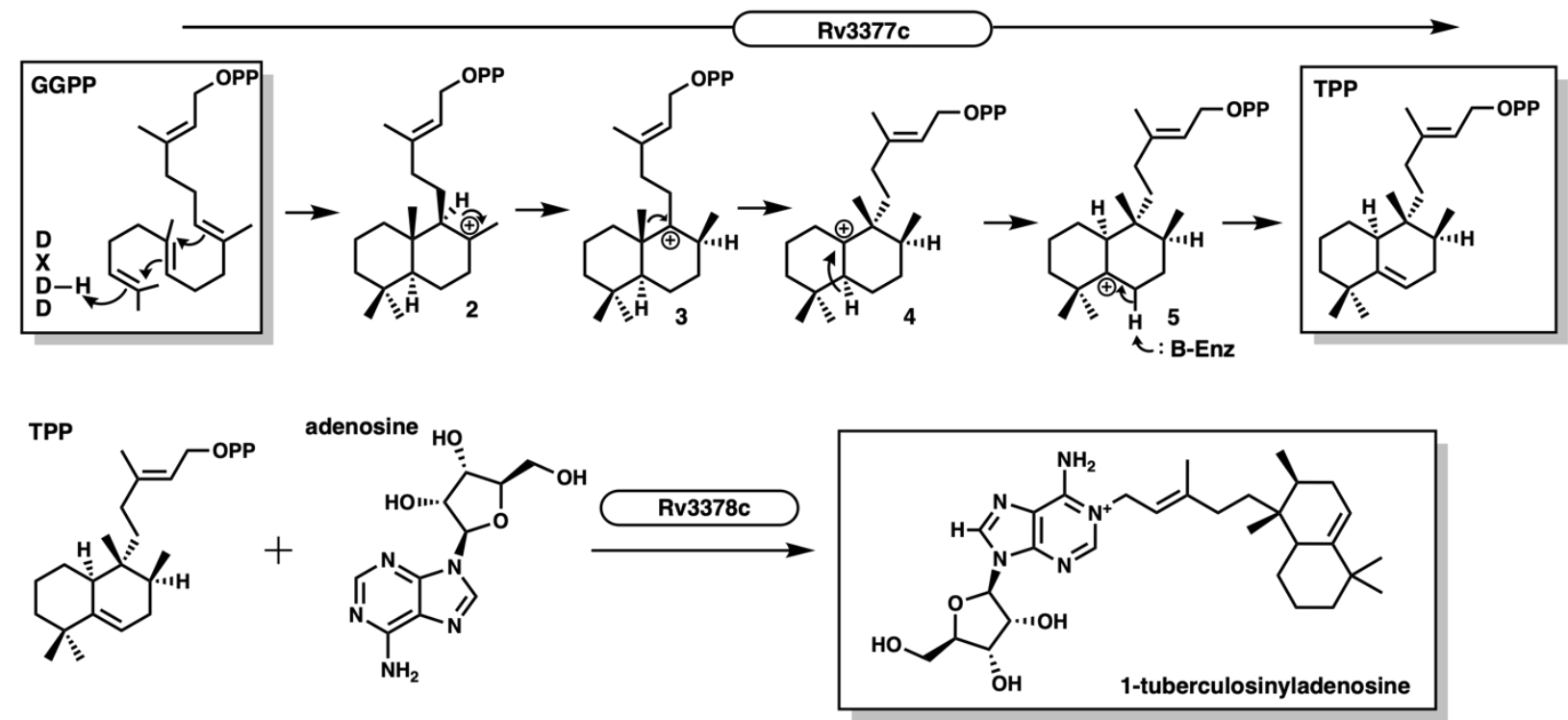

Proc. Natl. Acad. Sci. U. S. A. 2014, 111 (8), 2978-2983.

\section{Rv3377c crystal structure}

1. Crystal structure determination

Table s1 Data collection and refinement statistics. Statistics for the highest-resolution shell are shown in parentheses.

\begin{tabular}{|c|c|}
\hline & Rv3377c \\
\hline Resolution range $(\AA)$ & $40.01-2.718(2.815-2.718)$ \\
\hline Space group & C 121 \\
\hline Unit cell(Lengths: Å, Angles: degrees) & 124.90076 .42850 .1239091 .64490 \\
\hline Unique reflections & $12605(1086)$ \\
\hline Completeness (\%) & $98.46(85.43)$ \\
\hline Wilson B-factor $\left(\AA^{2}\right)$ & 47 \\
\hline Reflections used in refinement & $12602(1085)$ \\
\hline Reflections used for R-free & $611(48)$ \\
\hline R-work & $0.2139(0.3017)$ \\
\hline R-free & $0.2392(0.3491)$ \\
\hline Number of non-hydrogen atoms & 3789 \\
\hline macromolecules & 3757 \\
\hline solvent & 32 \\
\hline Protein residues & 483 \\
\hline RMS deviation, bonds (Å) & 0.005 \\
\hline
\end{tabular}




\begin{tabular}{|l|l|}
\hline RMS deviation, angles (degrees) & 0.97 \\
\hline Ramachandran favored (\%) & 97.90 \\
\hline Ramachandran allowed (\%) & 2.10 \\
\hline Ramachandran outliers $(\mathbf{\%})$ & 0.00 \\
\hline Rotamer outliers $(\mathbf{\%})$ & 0.26 \\
\hline Clashscore & 2.29 \\
\hline Average $\mathbf{B}-$ factor $\left(\AA^{2}\right)$ & 46 \\
\hline macromolecules $\left(\AA^{2}\right)$ & 46 \\
\hline solvent $\left(\AA^{2}\right)$ & 40 \\
\hline
\end{tabular}

2. Interdomain loop

An extended loop between the last two helices extends away from the $\beta$ domain, packs against the interdomain connections and inserts into a cleft in the $\gamma$ domain.

\section{$\beta$ domain}

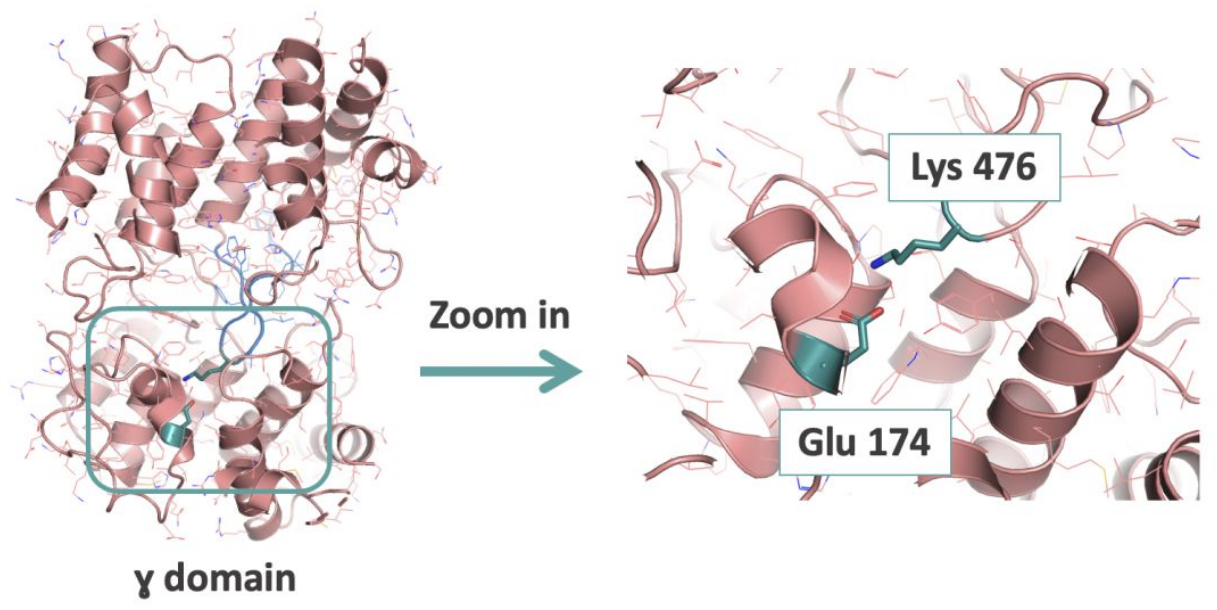

Figure s2. Lys476 at the tip of the loop forms an ion pair with Glu174 in the $\gamma$ domain, indicated in green colored residues. The interdomain loop is indicated in blue, with the conserved ion pair at the tip.

\section{Conserved QW motif}

Rv3377c contains two QW motif, which has the general consensus of $\mathrm{QX}_{2-5} \mathrm{GXW}$. One QW motif is in the $\beta$ domain and the other is in the $\gamma$ domain. Both QWmotifs has Gln and Trp stack, as shown in sticks. 


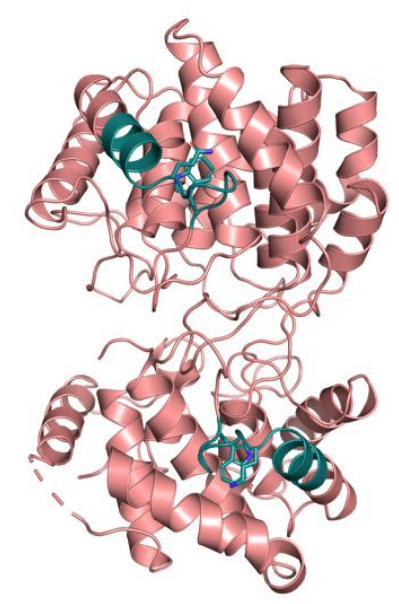

Figure s3 QW motif of Rv3377c, highlighted in green. There are two QW motifs present in Rv3377c, with Gln and Trp stack highlighted in stick.

Electronic structure calculation (Density functional theory)

1. Energies of intermediates and transition state structures

Table s2. Relative energies of intermediate and transition state structures

\begin{tabular}{|c|c|c|c|c|}
\hline Stationary Point & Energy & Hartrees & Kcal/mol & Relative Enery to 2 (kcal/mol) \\
\hline \multirow{2}{*}{$\mathbf{2}$} & HF & -626.8295054 & -393341.7829 & 0 \\
& Free Energy & -626.449601 & -393103.3891 & 0 \\
B & HF & -626.8183586 & -393334.7882 & 6.994728468 \\
& Free Energy & -626.441195 & -393098.1143 & 5.27484906 \\
3 & HF & -626.8243624 & -393338.5556 & 3.22728393 \\
& Free Energy & -626.444943 & -393100.4662 & 2.92294158 \\
C & HF & -626.8206499 & -393336.226 & 5.556914805 \\
& Free Energy & -626.439636 & -393097.136 & 6.25313715 \\
4 & HF & -626.8301962 & -393342.2164 & -0.433483908 \\
& Free Energy & -626.451723 & -393104.7207 & -1.33157622 \\
D & HF & -626.828109 & -393340.9067 & 0.876254964 \\
& Free Energy & -626.450468 & -393103.9332 & -0.54405117 \\
5 & HF & -626.8299773 & -393342.0791 & -0.296121969 \\
& Free Energy & -626.451546 & -393104.6096 & -1.22050695 \\
\hline
\end{tabular}

2. Intermediates and transition state structures

Intermediates and transition state structures are optimized with DFT mpw1pw91/6-31+g(d,p) level of theory. Optimized structures coordination are shown below. These files are saved as mol2 files, attached in "QM/Optimized_confs_methyl_ver" folder.

2 coordination mpw $1 \mathrm{pw} 91 / 6-31+\mathrm{g}(\mathrm{d}, \mathrm{p})$

C1 $-0.4259-1.9202 \quad-0.1761 \mathrm{C}$

$\begin{array}{llll}2 \mathrm{C} 2 & 0.1160 & 0.5211 & 0.2693 \mathrm{C}\end{array}$

$3 \mathrm{C} 3 \quad 1.8557 \quad-1.1155-0.0492 \mathrm{C}$ 


\begin{tabular}{|c|c|c|c|}
\hline $4 \mathrm{C} 4$ & & & \\
\hline & & & \\
\hline & & -2.6241 & \\
\hline & & & \\
\hline & & -3.1620 & \\
\hline & -0.2616 & 1.9575 & \\
\hline $10 \mathrm{C} 10$ & -2.3774 & -0.2225 & \\
\hline & & 1.2523 & \\
\hline & & & \\
\hline & 0.3 & 2.67 & \\
\hline & & & \\
\hline & & 2.10 & \\
\hline & & & \\
\hline & & & \\
\hline & & & \\
\hline & & & \\
\hline & & & \\
\hline & & & \\
\hline & & & \\
\hline & & & \\
\hline & & & \\
\hline & & & \\
\hline & & & \\
\hline & & & \\
\hline & & & \\
\hline & & & \\
\hline & & & \\
\hline & & & \\
\hline & & & \\
\hline & & & \\
\hline & 482 & -0. & \\
\hline & & & \\
\hline & & & \\
\hline & & & \\
\hline & & & \\
\hline & & & \\
\hline & & & \\
\hline & 3.9783 & 1.0759 & \\
\hline & & & \\
\hline & & 0.1324 & \\
\hline & & 1.0777 & \\
\hline $45 \mathrm{H} 45$ & -3.7012 & 1.4776 & $5-0.2824 \mathrm{H}$ \\
\hline \multicolumn{4}{|c|}{ coordination mpw1pw91/6-31+g(d,p) } \\
\hline & & & \\
\hline & & 0.4949 & 0.364 \\
\hline & 1.9729 & -1.1254 & $-0.3326 \mathrm{C}$ \\
\hline
\end{tabular}




\begin{tabular}{|c|c|c|c|}
\hline 4 & & & \\
\hline & -0.8352 & & \\
\hline & & -2.7039 & \\
\hline & & & \\
\hline & & -2.4952 & \\
\hline & & 1.9308 & \\
\hline $10 \mathrm{C} 10$ & -2.3160 & -0.2782 & -( \\
\hline & & & \\
\hline & & & \\
\hline & 5 & & \\
\hline & -1.9 & & \\
\hline & -0.0 & 2.06 & \\
\hline & & & \\
\hline & -2.2 & & \\
\hline & & & \\
\hline & 0 & & \\
\hline & & & \\
\hline & -0.8 & & \\
\hline & & & \\
\hline & & & \\
\hline & -2.7 & & \\
\hline & & & \\
\hline & -2.9 & & \\
\hline & & & \\
\hline & -2.4 & & \\
\hline & & & \\
\hline & & -1. & \\
\hline & 3 & -2. & \\
\hline & & -0 . & \\
\hline & & -1.5 & \\
\hline & -0.7 & -0.5 & \\
\hline & & & \\
\hline & -0.5 & -0.5 & \\
\hline & & -0.9 & \\
\hline & & & \\
\hline & & & \\
\hline & & 0.7 & \\
\hline & 3.04 & 1.84 & \\
\hline & & & \\
\hline & 3.8460 & 2.5619 & \\
\hline & 3.4266 & 1.0921 & \\
\hline $45 \mathrm{H} 45$ & -3.6268 & 1.4027 & -0.631 \\
\hline \multicolumn{4}{|c|}{ coordination mpw1pw91/6-31+g(d,p) } \\
\hline & & $-1.9849-$ & \\
\hline & 0.0385 & 0.5395 & \\
\hline & 1.9740 & -1.0261 & \\
\hline
\end{tabular}




\begin{tabular}{|c|c|c|c|}
\hline $4 \mathrm{C} 4$ & 1.0845 & -2.1951 & $0.0913 \mathrm{C}$ \\
\hline $5 \mathrm{H} 5$ & -1.0240 & -2.5117 & $0.3947 \mathrm{H}$ \\
\hline $6 \mathrm{H} 6$ & -0.5616 & -2.4398 & $-1.2812 \mathrm{H}$ \\
\hline $7 \mathrm{H} 7$ & 1.4556 & -3.1103 & $-0.3766 \mathrm{H}$ \\
\hline $8 \mathrm{H} 8$ & 1.1721 & -2.3605 & $1.1700 \mathrm{H}$ \\
\hline 9 C9 & -0.4806 & 1.9212 & $0.2454 \mathrm{C}$ \\
\hline $10 \mathrm{C} 10$ & -2.3844 & $\begin{array}{ll}4 & -0.3268\end{array}$ & $-0.1862 \mathrm{C}$ \\
\hline $11 \mathrm{C} 11$ & -2.7547 & 1.1369 & $-0.4645 \mathrm{C}$ \\
\hline $12 \mathrm{C} 12$ & -1.9839 & 2.1068 & $0.4133 \mathrm{C}$ \\
\hline $13 \mathrm{H} 13$ & 0.0901 & 2.4535 & $1.0108 \mathrm{H}$ \\
\hline $14 \mathrm{H} 14$ & -2.2361 & 3.1400 & $0.1634 \mathrm{H}$ \\
\hline $15 \mathrm{H} 15$ & -0.1477 & 2.3987 & $-0.6944 \mathrm{H}$ \\
\hline $16 \mathrm{H} 16$ & -2.2548 & 1.9750 & $1.4643 \mathrm{H}$ \\
\hline 17 H17 & -2.5693 & 1.3641 & $-1.5231 \mathrm{H}$ \\
\hline $18 \mathrm{C} 18$ & 1.4284 & 0.1611 & $9168 \mathrm{C}$ \\
\hline 19 H19 & 0.6217 & -0.4739 & $2893 \mathrm{H}$ \\
\hline $20 \mathrm{H} 20$ & 2.3728 & -0.2922 & $2.2257 \mathrm{H}$ \\
\hline $21 \mathrm{H} 21$ & 1.3460 & 1.1413 & $2.3867 \mathrm{H}$ \\
\hline $22 \mathrm{C} 22$ & -2.7796 & -0.7107 & $1.2484 \mathrm{C}$ \\
\hline $23 \mathrm{H} 23$ & -2.6586 & -1.7792 & $372 \mathrm{H}$ \\
\hline $24 \mathrm{H} 24$ & -2.2027 & -0.1722 & $.0057 \mathrm{H}$ \\
\hline $25 \mathrm{H} 25$ & -3.8352 & -0.4781 & $1.4110 \mathrm{H}$ \\
\hline $26 \mathrm{C} 26$ & -3.1433 & -1.2164 & $-1.1777 \mathrm{C}$ \\
\hline 27 H27 & -4.2177 & -1.0451 & $-1.0735 \mathrm{H}$ \\
\hline $28 \mathrm{H} 28$ & -2.8731 & -0.9887 & $-2.2140 \mathrm{H}$ \\
\hline 29 H29 & -2.9684 & -2.2801 & $-1.0044 \mathrm{H}$ \\
\hline 30 C30 & 3.4427 & -1.3469 & $-0.0421 \mathrm{C}$ \\
\hline $31 \mathrm{H} 31$ & 3.7085 & -2.2697 & $-0.5628 \mathrm{H}$ \\
\hline 32 H32 & 4.1234 & -0.5717 & $-0.3973 \mathrm{H}$ \\
\hline $33 \mathrm{H} 33$ & 3.6422 & -1.5151 & $1.0199 \mathrm{H}$ \\
\hline 34 C34 & -0.8472 & -0.5191 & $-0.3845 \mathrm{C}$ \\
\hline $35 \mathrm{C} 35$ & 1.4828 & 0.2922 & $0.3450 \mathrm{C}$ \\
\hline 36 H36 & -0.6472 & -0.1906 & $-1.4463 \mathrm{H}$ \\
\hline H37 & 1.8664 & $-0.8 \varepsilon$ & $-1.3993 \mathrm{H}$ \\
\hline $38 \mathrm{C} 38$ & 2.4037 & 1.4862 & $-0.0014 \mathrm{C}$ \\
\hline 39 H39 & 2.0454 & 2.3929 & $0.4918 \mathrm{H}$ \\
\hline $40 \mathrm{H} 40$ & 3.3718 & 1.2806 & $0.4625 \mathrm{H}$ \\
\hline $41 \mathrm{C} 41$ & 2.5990 & 1.7552 & $-1.4911 \mathrm{C}$ \\
\hline $42 \mathrm{H} 42$ & 1.6548 & 1.9213 & $-2.0206 \mathrm{H}$ \\
\hline $43 \mathrm{H} 43$ & 3.2031 & 2.6544 & $-1.6280 \mathrm{H}$ \\
\hline $44 \mathrm{H} 44$ & 3.1182 & 0.9377 & $-1.9952 \mathrm{H}$ \\
\hline $45 \mathrm{H} 45$ & $5 \quad-3.8300$ & $\begin{array}{ll}0 & 1.2653\end{array}$ & $-0.3109 \mathrm{H}$ \\
\hline \multicolumn{4}{|c|}{ oordination mpw1pw91/6-31+g(d,p) } \\
\hline $1 \mathrm{C} 1$ & -0.3721 & -1.9204 & $-0.3762 \mathrm{C}$ \\
\hline $2 \mathrm{C} 2$ & 0.0228 & 0.5762 & $-0.1061 \mathrm{C}$ \\
\hline $\mathrm{C} 3$ & 1.9888 & -1.0213 & $-0.3019 \mathrm{C}$ \\
\hline
\end{tabular}




\begin{tabular}{|c|c|c|c|}
\hline & & & \\
\hline & -1.0734 & & \\
\hline & -0.4907 & -2.0674 & \\
\hline & 1.4091 & & \\
\hline & 1.1158 & -2.42 & \\
\hline & -0.5222 & 1.9669 & \\
\hline & -2.3698 & -0.3550 & \\
\hline & -2.7523 & & $119 \mathrm{C}$ \\
\hline & -2.0 & & \\
\hline & -0.0 & & \\
\hline & -2.3 & & \\
\hline & -0.1 & & \\
\hline & & & \\
\hline & -2.5 & & \\
\hline & & & \\
\hline & $0.7^{\prime}$ & & \\
\hline & & & \\
\hline & 1.2 & & \\
\hline & -2.7 & & \\
\hline & -2.6 & & \\
\hline & -2.1 & & \\
\hline & -3.7 & & \\
\hline & -3.1 & & \\
\hline & -4.1 & & \\
\hline & -2.8 & & $89 \mathrm{H}$ \\
\hline & & & \\
\hline & & & \\
\hline & 3.7 & & \\
\hline & & & \\
\hline & 3.5902 & & \\
\hline & -0.8 & & \\
\hline & & & \\
\hline & 0.0 & & \\
\hline & 1.92 & & \\
\hline & 2.39 & & -0 . \\
\hline & 2.02 & & \\
\hline & & & \\
\hline & 2.5706 & & $-1.4964 \mathrm{C}$ \\
\hline & & & \\
\hline & 3.1715 & & $-1.6370 \mathrm{H}$ \\
\hline & & & \\
\hline $5 \mathrm{H} 45$ & -3.8370 & 1.1920 & $-0.4104 \mathrm{I}$ \\
\hline
\end{tabular}

TS B coordination mpwlpw91/6-31+g(d,p)

$\begin{array}{llll}1 \mathrm{C} 1 & 0.4270 & 1.9639 & 0.2795 \mathrm{C}\end{array}$

$\begin{array}{llll}2 \mathrm{C} 2 & -0.1262 & -0.5030 & 0.4146 \mathrm{C}\end{array}$

$\begin{array}{llll}3 \mathrm{C} 3 & -1.9785 & 1.1692 & -0.1153 \mathrm{C}\end{array}$ 


\begin{tabular}{|c|c|c|c|}
\hline & & & \\
\hline & & 2.0021 & \\
\hline & & .7203 & \\
\hline & & & \\
\hline & -1.4229 & 3.0629 & \\
\hline & & & \\
\hline & 2.3201 & 0.2762 & \\
\hline & & & \\
\hline & 1.7034 & -2.2 & \\
\hline & -0.3 & & \\
\hline & & -3 . & \\
\hline & -0.06 & -1.8 & \\
\hline & & & \\
\hline & & -1 . & \\
\hline & & & \\
\hline & & & \\
\hline & & & \\
\hline & & -0 . & \\
\hline & & & \\
\hline & & & \\
\hline & & & \\
\hline & & & \\
\hline & & & \\
\hline & & & \\
\hline & & & \\
\hline & & & \\
\hline & & & \\
\hline & & & \\
\hline & & & \\
\hline & & & \\
\hline & & & \\
\hline & & & \\
\hline & & & \\
\hline & & & \\
\hline & & & \\
\hline & & -2.0 & \\
\hline & & & \\
\hline & & -1.8361 & $98 \mathrm{C}$ \\
\hline & & & \\
\hline & -3.9045 & -2.5699 & $-0.7663 \mathrm{H}$ \\
\hline & & -1.0836 & \\
\hline $45 \mathrm{H} 45$ & 3.6060 & -1.3977 & $-0.7205 \mathrm{H}$ \\
\hline \multicolumn{4}{|c|}{ C coordination mpwlpw91/6-31+g(d,p) } \\
\hline & & -1.9267 & \\
\hline & 0.1238 & 0.5556 & $0.0135 \mathrm{C}$ \\
\hline & 2.0094 & -1.1326 & $-0.2387 \mathrm{C}$ \\
\hline
\end{tabular}




\begin{tabular}{|c|c|c|c|}
\hline & 508 & 001 & \\
\hline & -1.1326 & -2.5720 & \\
\hline & -0.3198 & -2.2449 & \\
\hline & & -3.1662 & \\
\hline & 0.8480 & -2.3012 & \\
\hline & -0.3783 & 1.9862 & \\
\hline $\mathrm{C} 10$ & -2.3504 & -0.2831 & -0 . \\
\hline & -2.7528 & & $20 \mathrm{C}$ \\
\hline & -1.8089 & 2.15 & \\
\hline & 0.29 & & \\
\hline & -2.1 & & \\
\hline & -0.34 & 2.23 & -1 . \\
\hline & & & \\
\hline & -2.7 & & -1 . \\
\hline & & & \\
\hline & -0.2210 & & \\
\hline & & & \\
\hline & 0.5936 & & \\
\hline & -2.63 & & \\
\hline & -2.2 & & \\
\hline & -2.2 & & \\
\hline & -3.7 & & \\
\hline & -3.2 & & \\
\hline & -4.2 & & \\
\hline & -3.0 & & \\
\hline & & & \\
\hline & & & \\
\hline & 3.61 & & \\
\hline & & & \\
\hline & 3.32 & & \\
\hline & -0.8 & -0. & \\
\hline & 1.50 & & \\
\hline & -0.8473 & -0.1 & $47 \mathrm{H}$ \\
\hline & & & \\
\hline & & & \\
\hline & 2.1477 & & \\
\hline & 3.37 & & \\
\hline & 3.0710 & 1.6926 & -1.2 \\
\hline & & & -1.8 \\
\hline & 3.7989 & & $-1.1672 \mathrm{H}$ \\
\hline & & & -1.70 \\
\hline 5 & -3.7729 & 1.3245 & $0.0827 \mathrm{H}$ \\
\hline
\end{tabular}

TS D coordination mpwlpw91/6-31+g(d,p)

$\begin{array}{llll}1 \mathrm{C} 1 & 0.3141 & -1.9534 & 0.2670 \mathrm{C}\end{array}$

$\begin{array}{llll}2 \mathrm{C} 2 & -0.0214 & 0.5329 & -0.1827 \mathrm{C}\end{array}$

$\begin{array}{llll}3 \mathrm{C} 3 & -2.0078 & -1.0080 & 0.2443 \mathrm{C}\end{array}$ 


\begin{tabular}{|c|c|c|c|}
\hline & & -2.1515 & \\
\hline & 1.0063 & -2.6192 & \\
\hline & 0.4054 & -2.2256 & \\
\hline & -1.4885 & -3.1007 & \\
\hline & -1.1341 & -2.2400 & $0 \mathrm{H}$ \\
\hline & 0.5319 & 1.9251 & -0.42 \\
\hline $\mathrm{C} 10$ & 2.3662 & -0.3755 & \\
\hline & 2.7490 & 1.1037 & \\
\hline & 2.0359 & & \\
\hline & -0.0053 & 2.3359 & \\
\hline & & & \\
\hline & 0.2436 & 2.5569 & $5 \mathrm{H}$ \\
\hline & & & \\
\hline$[17$ & 2.5119 & & $7 \mathrm{H}$ \\
\hline $\mathrm{C} 18$ & -1.79 & 0.39 & -1.8 \\
\hline & & & \\
\hline & -2.8610 & 0.26 & -2.0 \\
\hline & & & \\
\hline $\mathrm{C} 22$ & 2.9590 & -0.9 & -1.1 \\
\hline & 2.8081 & & \\
\hline & 2.5463 & -0.4 & -2. \\
\hline & & & \\
\hline & 2.93 & & \\
\hline 27 & 4.010 & & 1.4 \\
\hline & & -0 . & \\
\hline 29 & 2.7901 & & 1.2 \\
\hline $\mathrm{C} 30$ & -3.4777 & 352 & -0 . \\
\hline & -3.7545 & -2 & $3 \mathrm{H}$ \\
\hline H32 & -4.1527 & 374 & \\
\hline & -3.6691 & & $7 \mathrm{H}$ \\
\hline & & & \\
\hline & -1.5390 & & $97 \mathrm{C}$ \\
\hline & & & \\
\hline 37 H37 & -1.8843 & -0.9260 & $2 \mathrm{H}$ \\
\hline & -2.2959 & 1.5287 & \\
\hline 39 H39 & -1.9795 & 2.4614 & $-0.1810 \mathrm{H}$ \\
\hline $40 \mathrm{H} 40$ & -3.3500 & 1.4244 & $0.0249 \mathrm{H}$ \\
\hline $41 \mathrm{C} 41$ & -2.1767 & 1.6600 & $1.8097 \mathrm{C}$ \\
\hline $42 \mathrm{H} 42$ & -1.1356 & 1.7044 & $2.1535 \mathrm{H}$ \\
\hline & -2.6523 & & \\
\hline $44 \mathrm{H} 44$ & -2.6654 & 0.8405 & $2.3409 \mathrm{H}$ \\
\hline $45 \mathrm{H} 45$ & 3.8342 & 1.1897 & $0.2536 \mathrm{H}$ \\
\hline
\end{tabular}




\section{Docking simulations}

1. Conformational search and optimization

All intermediates are subjected to conformational search using Spartan 10 with the MMFF force field. All conformers generated were then fully optimized using Gaussian09 at the $\omega \mathrm{B} 97 \mathrm{XD} / 6-31+\mathrm{G}(\mathrm{d}, \mathrm{p})$ level of theory. Resulting structures within $5 \mathrm{kcal} / \mathrm{mol}$ of the lowest energy conformer for each intermediate were kept. Optimized conformers are attached in "QM/Optimized_confs_methyl_ver" folder.

Table s3. Energies of conformers for each intermediate. Lowest energies of each intermediate are highlighted as green.

\begin{tabular}{|c|c|c|c|}
\hline Intermediate & Conformer & Free Energy (a.u.) & Relative Energy (kcal/mol) \\
\hline 2 & 1 & -587.141432 & 0 \\
\hline \multirow{3}{*}{3} & 2 & -587.135394 & 3.78890538 \\
& 2 & -587.139064 & 0 \\
\multirow{4}{*}{4} & 1 & -587.136912 & 1.35040152 \\
& 4 & -587.145279 & 0 \\
& 2 & -587.14472 & 0.35077809 \\
& 3 & -587.143416 & 1.16905113 \\
& 1 & -587.141564 & 2.33119965 \\
& 1 & -587.14401 & 0 \\
& 3 & -587.143204 & 0.50577306 \\
& 2 & -587.140632 & 2.11972878 \\
& 4 & -587.139566 & 2.78865444 \\
\hline
\end{tabular}

\section{Docking simulations}

\section{a. Input files and output files of Rosetta}

Input files and output files of Rosetta are attached in SI "Docking" folder. The full ligand version of intermediate 2 are attached in "2_int_flig" folder.

Structures shown in figure 3 are in "Docking/Results" folder.

\section{b. Truncated structure and Constraint setup}

The first step of the reaction mechanism is proton donation from Asp295 to the substrate to generate 2. In the docking simulation, carbon 7 was constrained to Asp295. The constraint values were summarized in table s4. Angles and dihedrals were also constrained between Asp295 and intermediates to reflect the protonation step.

Table s4. Docking constraints setup

\begin{tabular}{|l|l|l|}
\hline \hline Constrained atoms & Type & Constraint value \\
\hline AspO-C7 & Distance & $2.5 \pm 0.5 \AA$ \\
\hline C6-C7-AspO & Angle & $90 \pm 10$ degrees \\
\hline
\end{tabular}




\begin{tabular}{|l|l|l|} 
C7-AspO-AspCG & Angle & $109 \pm 10$ degrees \\
\hline C7-AspO-AspCG-AspCB & Torsion & $0 \pm 20$ degrees \\
\hline
\end{tabular}
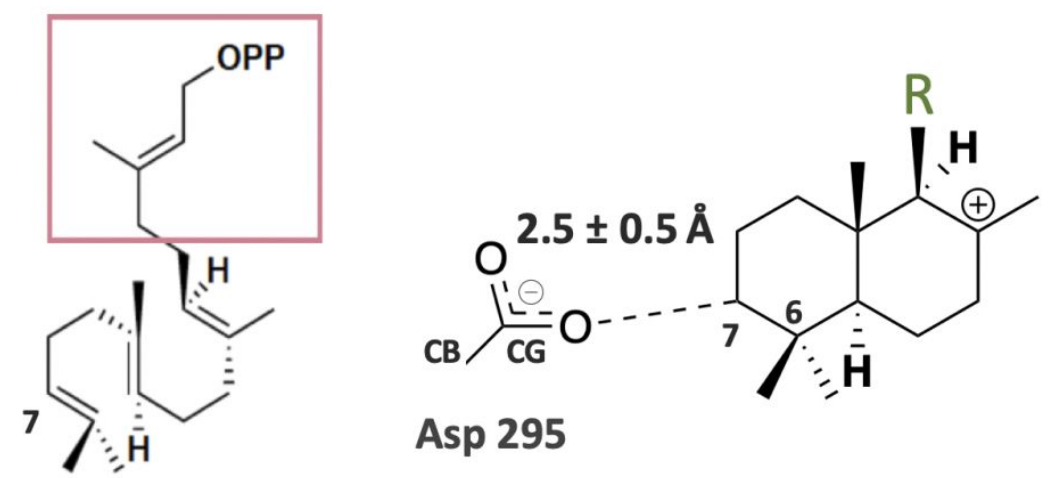

Asp 295

Figure s4. Left: truncated structure. Structure in red is substituted by $\mathrm{CH}_{3}$. Right: Constraint setup. The Asp295 is constraint to carbon 7 (protonated carbon).

\section{c. RMSD of docked structures}

The RMSD calculation are performed on each carbon in the carbocation skeleton between intermediates 2, 3, 4 and $\mathbf{5}$. The transition from 2-4 and 2-5 are higher possibly due to the multiple methyl group shifts. Lines and numbers shown on the plot represents the RMSD of the selected model shown in main text figure 3 . The RMSD of intermediate $2-3$ is 0.377 , RMSD of intermediate $2-4$ is 1.82 , RMSD of intermediate $2-5$ is 1.65 (see figure 3 for overlap of the intermediates).

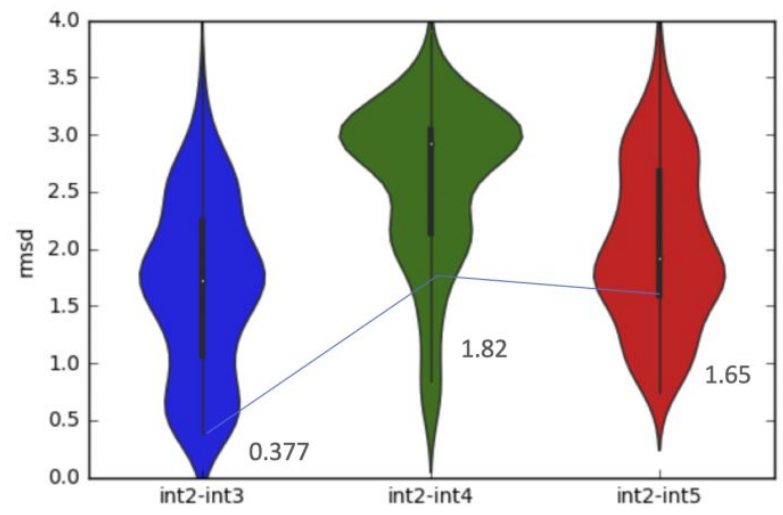

Figure s5. Violin plots of the RMSD calculations between each intermediate to 2. Dot represents mean value. Thick black line represents first quartile, thick line represents standard deviation. Lines and numbers shown on the plot represents the RMSD of the selected model shown figure 3. 


\section{Comparison with AtCPS}

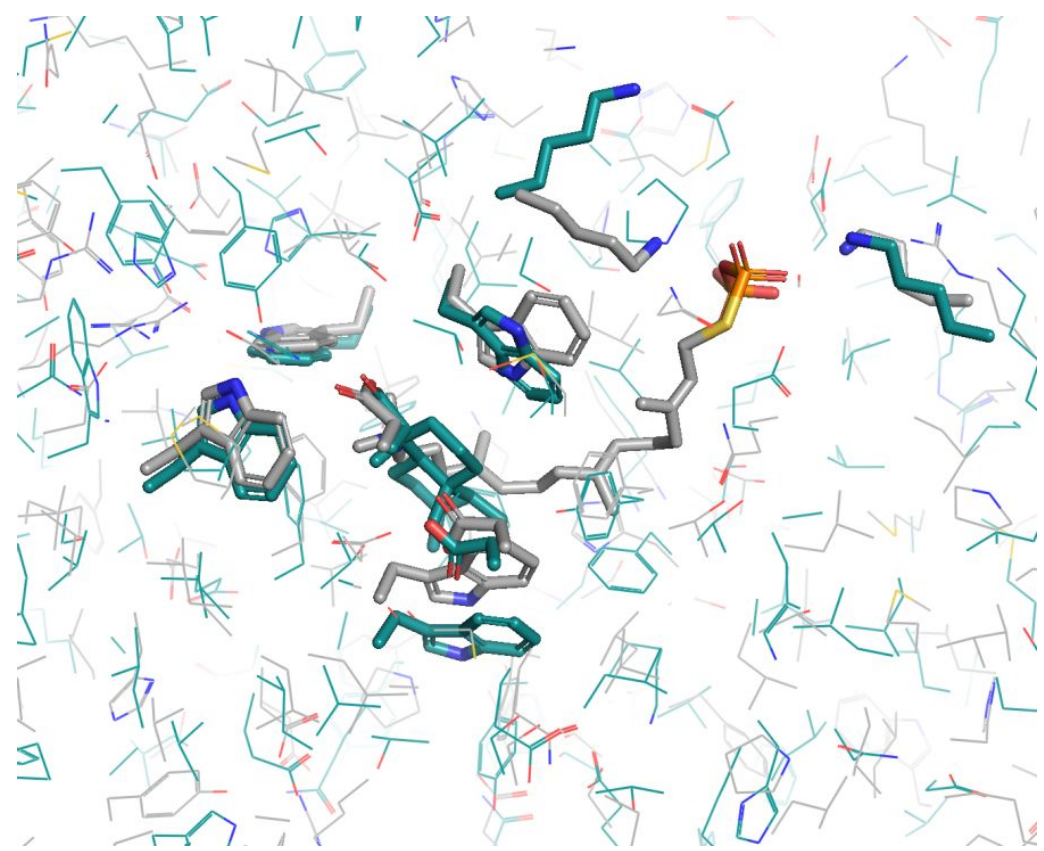

Figure s6. Comparison between Rv3377c and AtCPS. Common residues are shown in sticks. Green: Rv3377c. Grey: AtCPS. Docked intermediate 2 shown in green sticks in the middle. Co-crystalized (S)-15-aza-14,15-dihydrogeranylgeranyl thiolodiphosphate in AtCPS shown in grey sticks.

Input and output files

All input and output files are attached in SI, including:

Crystal structure

Pdb file and mtz file are attached in "Crystal_structure" folder.

QM calculation result

The intermediates and TSS are found with DFT method, these structures are attached in "Intermediates and TSS" folder.

Optimized conformers (truncated) for docking are attached in "Optimized_confs_methyl_ver".

Docking result

All Rosetta docking input and output files are attached in "Docking" folder. Each subfolder contains one intermediate result indicated by the folder name. Results shown in main text figure are attached in "Results" folder.

These files are also uploaded in this Github link:

https://github.com/siegel-lab-

ucd/Publication_Tiffy/tree/master/Crystal $\% 20$ structure $\% 20$ and $\% 20$ docking $\% 20$ study $\% 20$ of $\% 20$ Rv3377c\%20the\%20Mycobacterium\%20tuberculosis\%20diterpene \%20cyclase 